\title{
Let-7a enhances the sensitivity of hepatocellular carcinoma cells to cetuximab by regulating STAT3 expression
}

This article was published in the following Dove Press journal:

OncoTargets and Therapy

28 November 2016

Number of times this article has been viewed

\author{
Fei Xue' \\ Yanhui Liu \\ Hongwei Zhang' \\ Yu Wen' \\ Lei Yan' \\ Qiang Tang' \\ Erhui Xiao' \\ Dongyi Zhang'
}

'Department of Hepatobiliary and Pancreatic Surgery, Henan Provincial People's Hospital, Zhengzhou University People's Hospital, Zhengzhou, People's Republic of China; 'Department of Hematology, Henan Provincial People's Hospital, Zhengzhou University People's Hospital, Zhengzhou, People's Republic of China
Correspondence: Fei Xue

Department of Hepatobiliary and Pancreatic Surgery, Henan Provincial People's Hospital, Zhengzhou University People's Hospital, 7 Weiwu Road, Jinshui, Zhengzhou, Henan, 410003, People's Republic of China

Tel +8637l 65580365

Email xuefeidoctor@I63.com
Background: Let-7 miRNAs are reported to play an inhibitory role in carcinogenesis, tumor progression, recurrence, and pluripotency of cancer. However, few studies have reported the relationship between let-7 and drug sensitivity, especially for let-7a (a subtype of let-7). This study aimed to investigate the function of let-7a in regulating the sensitivity of hepatocellular carcinoma (HCC) cell lines to cetuximab.

Methods: The cytotoxicity of cetuximab on HCC cell lines (Huh7, Hep3B, HepG2, SNU449, and SNU387) was evaluated using a cell viability assay (the Cell Counting Kit- 8 assay) and a cell proliferation assay (the Click-iT EdU Imaging Kit) in the presence of a control, a let-7a mimic, and a let-7a inhibitor. Small interfering RNA to knockdown the expression of signal transducer and activator of transcription 3 (STAT3) were employed. Protein and mRNA expression levels were determined using quantitative polymerase chain reaction and Western blot analysis.

Results: It was found that let-7a enhances the sensitivity of HCC cells with an epithelial phenotype (Huh7, Hep3B, and HepG2) to cetuximab, but has no effect on cells with the mesenchymal phenotype (SNU449 and SNU387). It was determined that STAT3 was a target mRNA of let-7a using TargetScan. Expression of STAT3 and let-7a mRNA were negatively correlated in HCC cell lines. Moreover, let-7a altered the protein and mRNA expression of STAT3. Furthermore, STAT3 knockdown enhanced the function of cetuximab on HCC cell lines with epithelial phenotypes, but not on HCC cell lines with mesenchymal phenotypes. Finally, a rescue experiment confirmed that let-7a affected the sensitivity of HCC cell lines to cetuximab by interacting with STAT3.

Conclusions: There is a functional link between let-7a and STAT3 in enhancing the sensitivity of HCC cells with an epithelial phenotype to cetuximab. Our results provide novel insight into new methodologies for combating HCC drug resistance.

Keywords: hepatocellular carcinoma, microRNA, STAT3, let-7a

\section{Introduction}

Hepatocellular carcinoma (HCC) is the sixth most common malignancy worldwide, and has persistently increasing rates of both incidence and mortality. ${ }^{1}$ Curative surgical procedures, such as tumor resection and liver transplantation, are not available for advanced HCC patients; instead, they can only turn to chemotherapeutic drugs to slow down the progress of the tumor. ${ }^{2}$ Currently, sorafenib is the only chemotherapy drug that is widely used in clinical applications as a first-line treatment for advanced HCC. ${ }^{3}$ However, there is increasing evidence of resistance to this drug. ${ }^{4}$ As such, identification of curative second-line treatments for advanced HCC has become extremely urgent.

While cetuximab, an epidermal growth factor receptor (EGFR) inhibitor, was shown to display satisfactory curative effect in patients with non-small cell lung cancer, ${ }^{5}$ 
many clinical trials have indicated its modest activity in advanced HCC, even though some patients show high EGFR expression. ${ }^{6,7}$ However, a recent study revealed that rapamycin or an miR-146a mimic could enhance cetuximab cytotoxicity on HCC cell lines. ${ }^{8,9}$ Therefore, cetuximab may be a promising second-line treatment for HCC in combination with some other complementary medicines, such as microRNAs (miRNAs).

MiRNAs, endogenous noncoding RNA molecules (18-25 nucleotides in length), negatively regulate the expression of numerous target genes. For the past few years, miRNA profiling studies have indicated that many miRNAs are abnormally expressed in HCC tissues and affect the initiation and progression of HCC. ${ }^{10,11}$ Chiefly, the miRNA let-7 plays a vital role in tumor suppression in many cancers, including esophageal squamous cell carcinoma, lung cancer, nasopharyngeal carcinoma, and prostate cancer. ${ }^{12-15}$ Although miRNA let-7 is known to correlate with poor prognosis of hepatitis B virus-related HCC patients, ${ }^{16}$ few studies have investigated its precise function in HCC.

Let-7 has multiple subtypes (a, b, c, d, e, f, and g); however, the let-7a subtype has been correlated with tumor proliferation and differentiation. ${ }^{17-19}$ Therefore, in this study, we investigated whether the let-7a subtype could increase the sensitivity of HCC cell lines to cetuximab, and aimed to unravel its mechanism of action.

\section{Materials and methods Cell culture}

HCC cell lines Huh7, Hep3B, HepG2, SNU449, and SNU387 were purchased from the Chinese Academy of Science Cell Bank (Shanghai, People's Republic of China). The ethics committee and institutional review board of the Chinese Academy of Science Cell Bank gave ethical approval for this study. The HepG2 cell line was maintained in Dulbecco's Modified Eagle's Medium (Thermo Fisher Scientific, Waltham, MA, USA), Huh7 and Hep3B were maintained in Minimum Essential Medium (Thermo Fisher Scientific), and SNU449 and SNU387 were maintained in RPMI 1640 (Thermo Fisher Scientific). All culture media were supplemented with 10\% fetal bovine serum (FBS), and cells were incubated at $37^{\circ} \mathrm{C}$ in a humidified atmosphere of $5 \% \mathrm{CO}_{2}$. Cetuximab was obtained from Merck (Darmstadt, German) and dissolved in doubledistilled water for the purpose of this research.

\section{RNA oligoribonucleotides and transfection}

The let-7a mimic, inhibitor, control, siRNA-signal transducer and activator of transcription 3 (STAT3), and negative control
siRNA were synthesized by Qiagen (Venlo, the Netherlands). Cells were transfected using lipofectamine 2000 (Thermo Fisher Scientific) according to manufacturer's instructions.

\section{Cell viability assay}

HCC cell lines (Huh7, Hep3B, HepG2, SNU449, and SNU387) were seeded at a density of 3,000 cells/well in 96-well plates. After the cells completely adhered to the well, the culture medium was replaced with media containing $10 \%$ FBS and a certain concentration of cetuximab $(0,500,1,000$, 1,500 , or $2,000 \mu \mathrm{g} / \mathrm{mL}$ ) and cultivated at $37^{\circ} \mathrm{C}, 5 \% \mathrm{CO}_{2}$ for 48 hours. Cell viability was subsequently measured using a Cell Counting Kit-8 (CCK-8, Dojindo Laboratories, Tokyo, Japan) after 1-2 hours, according to manufacturer's instructions. An MRX II microplate reader (Dynex, Chantilly, VA, USA) was used to measure the optical density at $450 \mathrm{~nm}$.

\section{Western blot}

HCC cell lines (Huh7, Hep3B, HepG2, SNU449, and SNU387) were seeded at a density of $2.0 \times 10^{5}$ cells/well in 6 -well plates. After the cells completely adhered to the well, the let-7a mimic, inhibitor, control, si-STAT3, and negative control siRNA were added to the media, respectively. After 48 hours, cells were washed with phosphate-buffered saline three times, and lysed in radio immunoprecipitation assay lysis buffer $(50 \mathrm{mM}$ Tris$\mathrm{HCl}, \mathrm{pH} 7.4 ; 150 \mathrm{mM} \mathrm{NaCl} ; 1 \%$ Triton X-100; 1\% sodium deoxycholate; $0.1 \%$ sodium dodecyl sulphate; and $1 \mathrm{mM}$ phenylmethylsulfonyl fluoride) for 2 hours on ice. Sample proteins were quantified using a Pierce bicinchoninic acid protein assay (Thermo Fisher Scientific). Equal amounts of proteins were separated by sodium dodecyl sulphate polyacrylamide gel electrophoresis, transferred to polyvinylidene difluoride membranes, and probed with the antibody of interest. The primary antibody (stat3) concentration was 1:1,000, and the secondary antibody concentration was 1:2,000. All antibodies were purchased from Abcam company (Cambridge, MA, USA).

\section{Cell proliferation assay}

The proliferation of HCC cell lines (Huh7, Hep3B, HepG2, SNU449, and SNU387) was determined using the Click-iT EdU Imaging Kit (Thermo Fisher Scientific), according to the manufacturer's protocol. Briefly, cells were treated with miRNA let-7a mimics, inhibitors, or control for 24 hours, then incubated with an half maximal inhibitory concentration $\left(\mathrm{IC}_{50}\right)$ (Huh7: 2,564 $\mu \mathrm{g} / \mathrm{mL}$, Hep3B: 2,299 $\mu \mathrm{g} / \mathrm{mL}$, HepG2: 7,167 $\mu \mathrm{g} / \mathrm{mL}, \mathrm{SNU} 449: 2,450 \mu \mathrm{g} / \mathrm{mL}$, and SNU387: $3,182 \mu \mathrm{g} / \mathrm{mL}$ ) of cetuximab for 24 hours, and finally with $10 \mu \mathrm{M}$ EdU for 2 hours before fixation, permeabilization, and EdU staining. Cell nuclei were stained with Hoechst 
33342 (Thermo Fisher Scientific) at a concentration of $5 \mu \mathrm{g} / \mathrm{mL}$ for 30 minutes.

\section{Real-time quantitative polymerase chain reaction}

Total RNA was extracted from HCC cell lines using TRIzol reagent following the manufacturer's protocol (Thermo Fisher Scientific). First-strand complementary DNA was generated using a Reverse Transcription System Kit (Promega Corporation, Madison, WI, USA). Real-time and polymerase chain reaction (PCR) primers for miRNA let-7a and STAT3 were purchased from TAKARA Biotechnology (Dalian, People's Republic of China). Expression of each gene was measured by quantitative PCR with the Applied Biosystems 7,500/7,500 Fast Real-Time PCR system (Thermo Fisher Scientific) and SYBR green dye (TAKARA Biotechnology). Glyceraldehyde 3-phosphate dehydrogenase and U6 were used as internal controls, and the $2^{-\Delta \Delta \mathrm{Ct}}$ method was used for relative quantification. All reactions were performed in triplicate.

\section{Statistical analysis}

SPSS17.0 software was used for statistical analysis. The experimental data were expressed as mean \pm standard deviation, and assessed by a two-tailed Student's $t$-test. A $P<0.05$ level of statistical significance was used.

\section{Results}

\section{Let-7a enhances the sensitivity of HCC cells with epithelial phenotypes to cetuximab}

To investigate the function of let-7a on the sensitivity of HCC cell lines to cetuximab, we used a cell viability assay (the CCK-8 assay) and a cell proliferation assay (the EdU assay). We detected the viability of HCC cell lines incubated in the presence of different cetuximab concentrations. Each cell line was divided into three groups: a let-7a mimic, a let-7a inhibitor, and a control group. Compared with the control, the let- $7 \mathrm{a}$ mimic enhanced the function of cetuximab in HCC cells with epithelial phenotypes (ie, Huh7, Hep3B, and HepG2), while the let-7a inhibitor displayed the opposite results (Figure 1A-C). However, no difference in the function of cetuximab in HCC cells with mesenchymal phenotypes (ie, SNU387 and SNU449) was observed (Figure 1D and E). Under the same condition, the EdU assay also revealed that the let-7a mimic could enhance the function of cetuximab on the proliferation of HCC cells with epithelial phenotypes but not in those with mesenchymal phenotypes (Figure 1A-E and Table 1).

\section{STAT3 is the target gene of let-7a and is negatively regulated by let-7a}

Recent studies have reported that STAT3 expression is correlated with the effect of anti-EGFR therapeutic drugs in solid tumors. ${ }^{20}$ Therefore, we hypothesized that STAT3 is regulated by the let-7a. We used TargetScan (www.targetscan.org) to predict the associated miRNAs for STAT3. As expected, STAT3 is a target gene of let-7a (Figure 2A). Then, we detected the expression of let-7a and STAT3 mRNA in the HCC cell lines (Huh7, Hep3B, HepG2, SNU387, and SNU449) by PCR. We found the mRNA expression of let-7a was negatively correlated with mRNA expression of STAT3 (Figure 2B). Next, we treated these HCC cell lines separately with a let-7a mimic, inhibitor, and control, and detected the expression of STAT3 and let-7a by PCR. Compared with the control, expression of STAT3 was suppressed in the presence of the let-7a mimic (Figure 2C). However, STAT3 was overexpressed in the presence of the let-7a inhibitor (Figure 2D). These changes in STAT3 expression were further confirmed by our Western blot analysis of STAT3 protein expression (Figure 2E). Therefore, our results suggest that STAT3 is the target gene of let-7a.

\section{A}

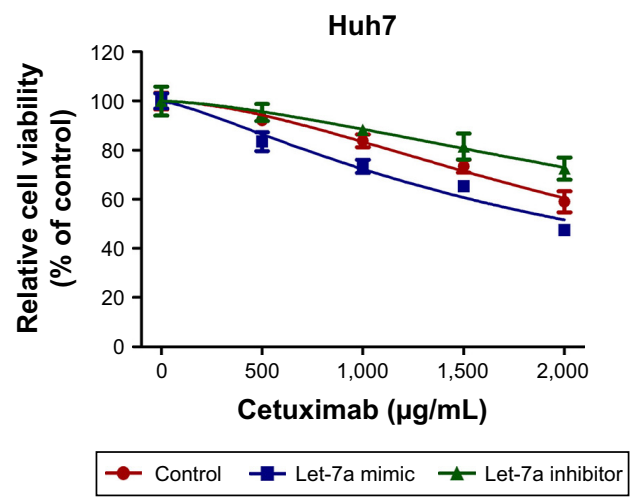

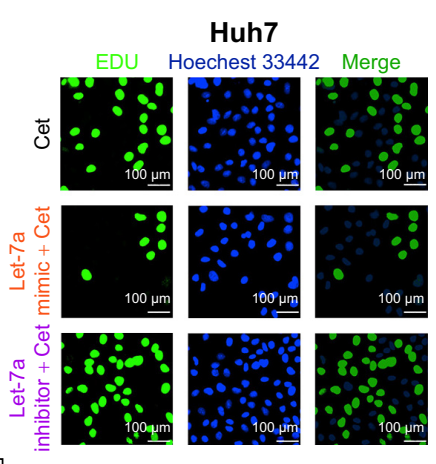

Huh7

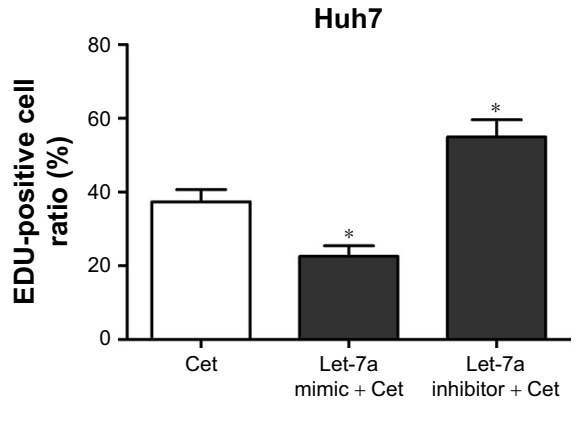

Figure I (Continued) 
B

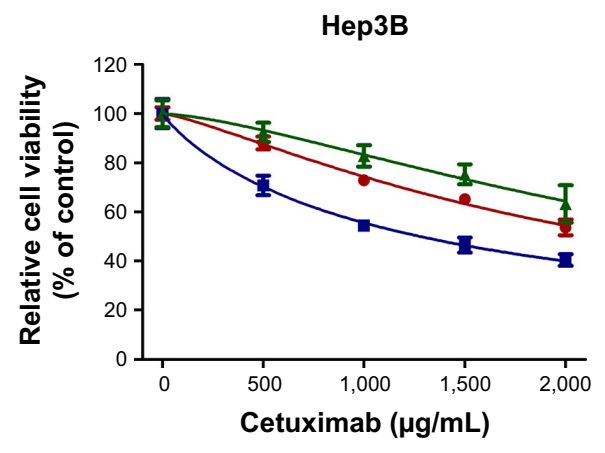

C

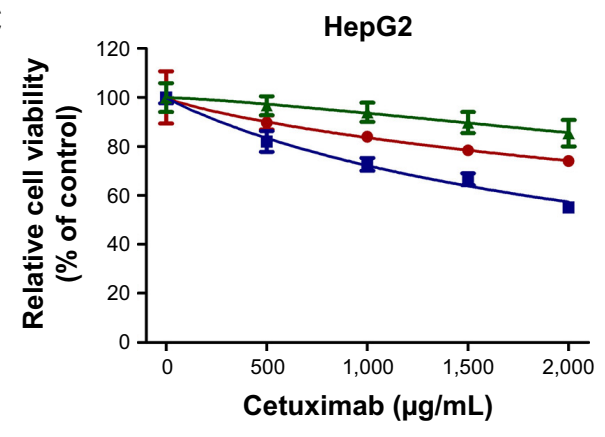

D

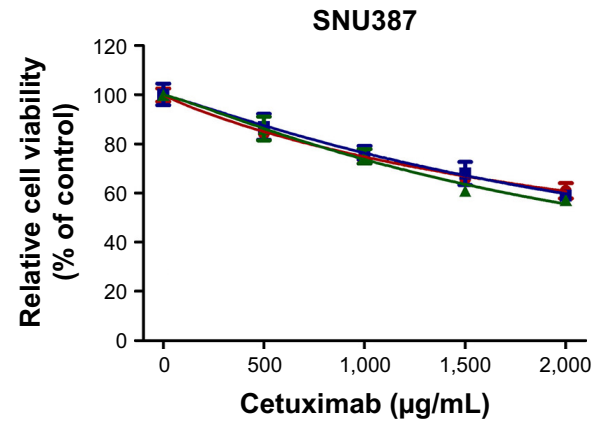

E

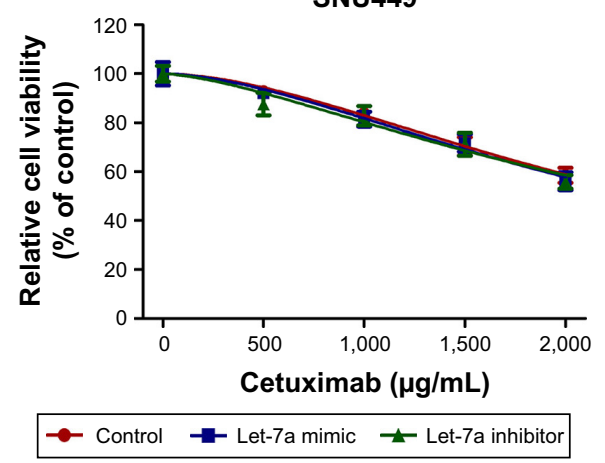

Hep3B

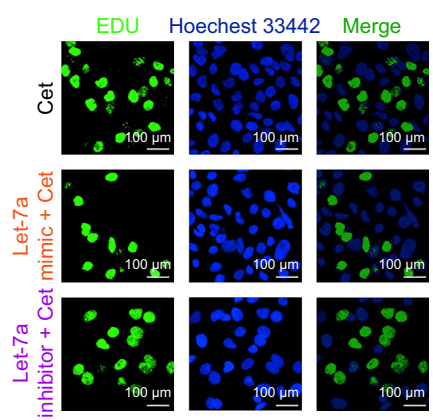

HepG2

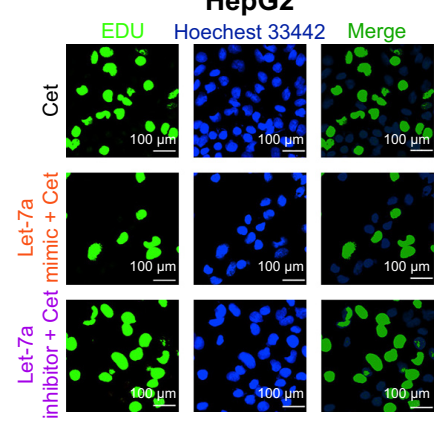

SNU387

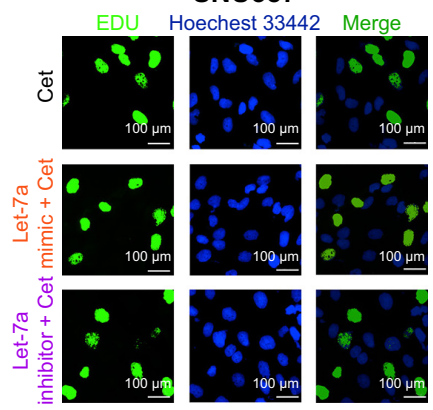

SNU449

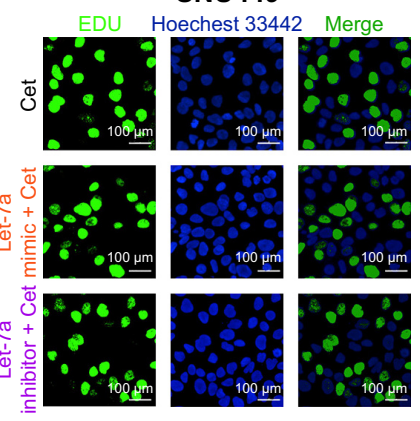

Нер3В

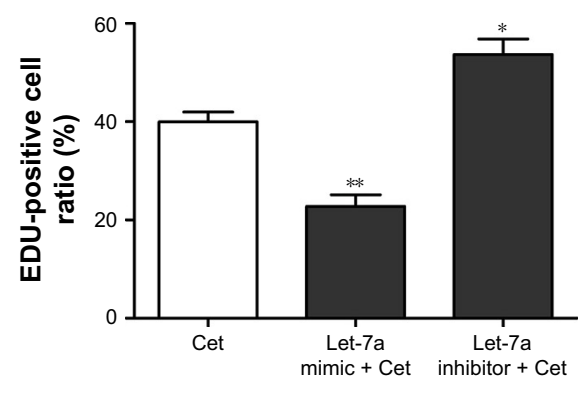

HepG2

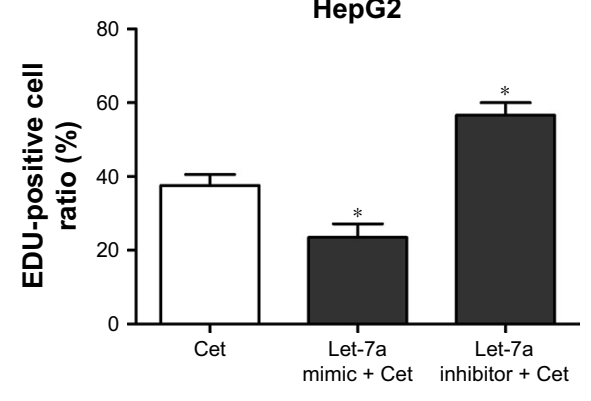

SNU387

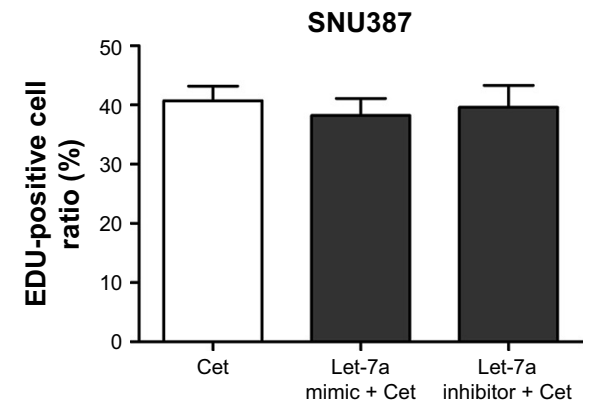

SNU449

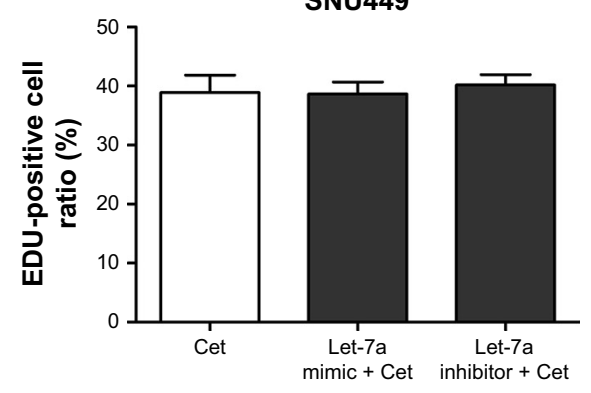

Figure I Let-7a can enhance the sensitivity of HCC cells with epithelial phenotypes to cetuximab.

Notes: (A-E) All HCC cell lines were separated into three groups: the let-7a mimic, inhibitor, and control groups. Cell viability was determined using the CCK-8 assay in the presence of different concentrations of cetuximab $(0,500,1,000,1,500$, and 2,000 $\mu \mathrm{g} / \mathrm{mL})$. Cell proliferation rate (using the EdU assay) was determined in the presence of

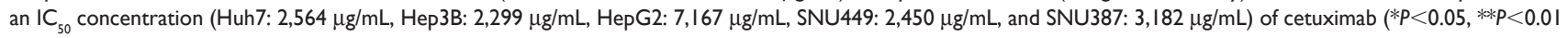
vs control. Huh7: $P_{\text {Let-7a mimic }+C e t}=0.0284, P_{\text {Let-7a inhibitor+Cet }}=0.0378$; Hep3B: $P_{\text {Let-7a mimictCet }}=0.005$ I, $P_{\text {Let-7a inhibitor+Cet }}=0.02$ I8; HepG2: $\left.P_{\text {Let-7a mimictCet }}=0.0415, P_{\text {Let-7a inhibitor+Cet }}=0.0138\right)$.

Abbreviations: CCK-8, Cell Counting Kit-8; Cet, cetuximab; $I_{50}$, half maximal inhibitory concentration; HCC, hepatocellular carcinoma.

Suppression of STAT3 could enhance the sensitivity of epithelial phenotype HCC cells to cetuximab

As STAT3 was negatively regulated by let-7a, and a let-7a mimic can enhance sensitivity of HCC cells with epithelial phenotypes to cetuximab, we hypothesized that the suppression of STAT3 could lead to the same result. To examine this, first we employed siRNA to knockdown the expression of STAT3 and verified the efficiency by Western blot (Figure 3A). Then, we used the CCK-8 assay to detect 
Table I IC ${ }_{50}$ values for cetuximab in HCC cell lines with or without let-7a mimic or let-7a inhibitor treatment

\begin{tabular}{llll}
\hline HCC cell line & $\mathbf{I C}_{50}(\mu \mathrm{g} / \mathrm{mL})$ & & Cetuximab + let-7a inhibitor \\
\cline { 2 - 4 } & Cetuximab & $2,104 \pm 96.5 I^{*}$ & $3,853 \pm 495.5^{*}$ \\
\hline Huh7 & $2,564 \pm 109.8$ & $1,279 \pm 44.54^{*}$ & $3,000 \pm 287.3^{*}$ \\
Hep3B & $2,299 \pm 76.91$ & $2,732 \pm 170.5^{*}$ & $7,913 \pm 3625^{*}$ \\
HepG2 & $7,167 \pm 2293$ & $2,814 \pm 216.9$ & $2,473 \pm 137.0$ \\
SNU387 & $3,182 \pm 190.9$ & $2,406 \pm 99.81$ & $2,511 \pm 157.8$ \\
SNU449 & $2,450 \pm 70.66$ &
\end{tabular}

Notes: $* P<0.05$ vs cetuximab alone. Data presented as mean \pm SD.

Abbreviations: $\mathrm{HCC}$, hepatocellular carcinoma; $\mathrm{IC}_{50}$, half maximal inhibitory concentration; $\mathrm{SD}$, standard deviation.

the cell viability of HCC cell lines in different cetuximab concentrations. Each cell line was divided into two groups: a STAT3 siRNA group and a negative siRNA group. Compared to the control, suppression of STAT3 enhanced the function of cetuximab on HCC cell lines with epithelial phenotypes (ie, Huh7, Hep3B, and HepG2), but not on HCC cell lines with mesenchymal phenotypes (ie, SNU387 and SNU449; Figure 3B-F and Table 2). Therefore, negative regulation of STAT3 is correlated with the increased sensitivity of HCC cells with epithelial phenotypes to cetuximab.

\section{STAT3 suppression impairs the role of let-7a on increasing the sensitivity of HCC cells to cetuximab}

To verify that let-7a enhances sensitivity of HCC cells with epithelial phenotype to cetuximab through negatively regulating STAT3 expression, we conducted a rescue experiment. We adopted siRNA to knockdown the expression of STAT3 in HCC cell lines. Then we used the CCK-8 assay to detect the cell viability of HCC cell lines in different cetuximab concentrations. Again, each cell line was divided into three groups (a let-7a mimic, a let-7a inhibitor, and a control group). All HCC cell lines showed the same result: that is, the regulatory ability of let-7a on the sensitivity of HCC cell lines to cetuximab was reversed when STAT3 was suppressed (Figure 4A-E). This indicates that let-7a enhances the sensitivity of HCC cell lines with epithelial phenotypes to cetuximab by regulating STAT3 expression.

\section{Discussion}

Traditional surgical treatment or liver transplantation are the only potentially curative therapies for HCC; however, in the case of advanced HCC, chemotherapy is the only available option..$^{1-3}$ Moreover, with the increase in resistance to first-line chemotherapy treatments (such as for sorafenib), ${ }^{4}$ the identification of curative second-line treatments for advanced $\mathrm{HCC}$ is urgently required. Furthermore, it has been determined that EGFR activation is a potential determinant of primary resistance of HCC cells to sorafenib, while inhibition of EGFR by its specific inhibitor cetuximab promotes the efficacy of sorafenib. ${ }^{20}$ So recent studies indicate that cetuximab may be a promising second-line drug for advanced HCC, although it only shows moderate activity. ${ }^{7-9}$ MiRNAs have shown some promise for enhancing cetuximab cytotoxicity on HCC cell lines. ${ }^{8,9}$ Therefore, we examined whether the well-known tumor suppressor, let-7a, ${ }^{12-15}$ could enhance the cytotoxicity of cetuximab in HCC cells. We found that exogenous let-7a overexpression could enhance the sensitivity of HCC cells with epithelial phenotypes to cetuximab, while no difference in sensitivity was observed on HCC cell lines with mesenchymal phenotypes.

Several recent studies have reported that anti-EGFR therapeutic efficacy correlates directly with inhibition of STAT3 activity. In particular, patients who develop cetuximab resistance always have overexpression of STAT3. ${ }^{21}$ Furthermore, a novel STAT3 inhibitor NSC 74859 enhances the antiproliferative activity of cetuximab in HCC..$^{22}$ Moreover, the activity of STAT3 was correlated with tumor proliferation, migration, and invasion. ${ }^{23}$ Cetuximab, an anti-EGFR monoclonal antibody that targets the extracellular domain of the EGFR, is used to treat several cancers. ${ }^{24}$ Furthermore, the previous research demonstrated that activation of STAT3 was shown to play a role in tumor resistance to anticancer drugs, and downregulation of pSTAT3 increased the sensitivity of human cancer cells to cetuximab. Therefore, we examined whether the function of let-7a on HCC sensitivity to cetuximab was related to STAT3. We used TargetScan to search the correlated miRNAs of the STAT3 3'-UTR segment, and found that STAT3 is a target gene of let-7a. Furthermore, we found that STAT3 expression was negatively correlated with let-7a. This suggests that STAT3 is negatively regulated by let-7a. Indeed, in the presence of a let-7a mimic, STAT3 expression 

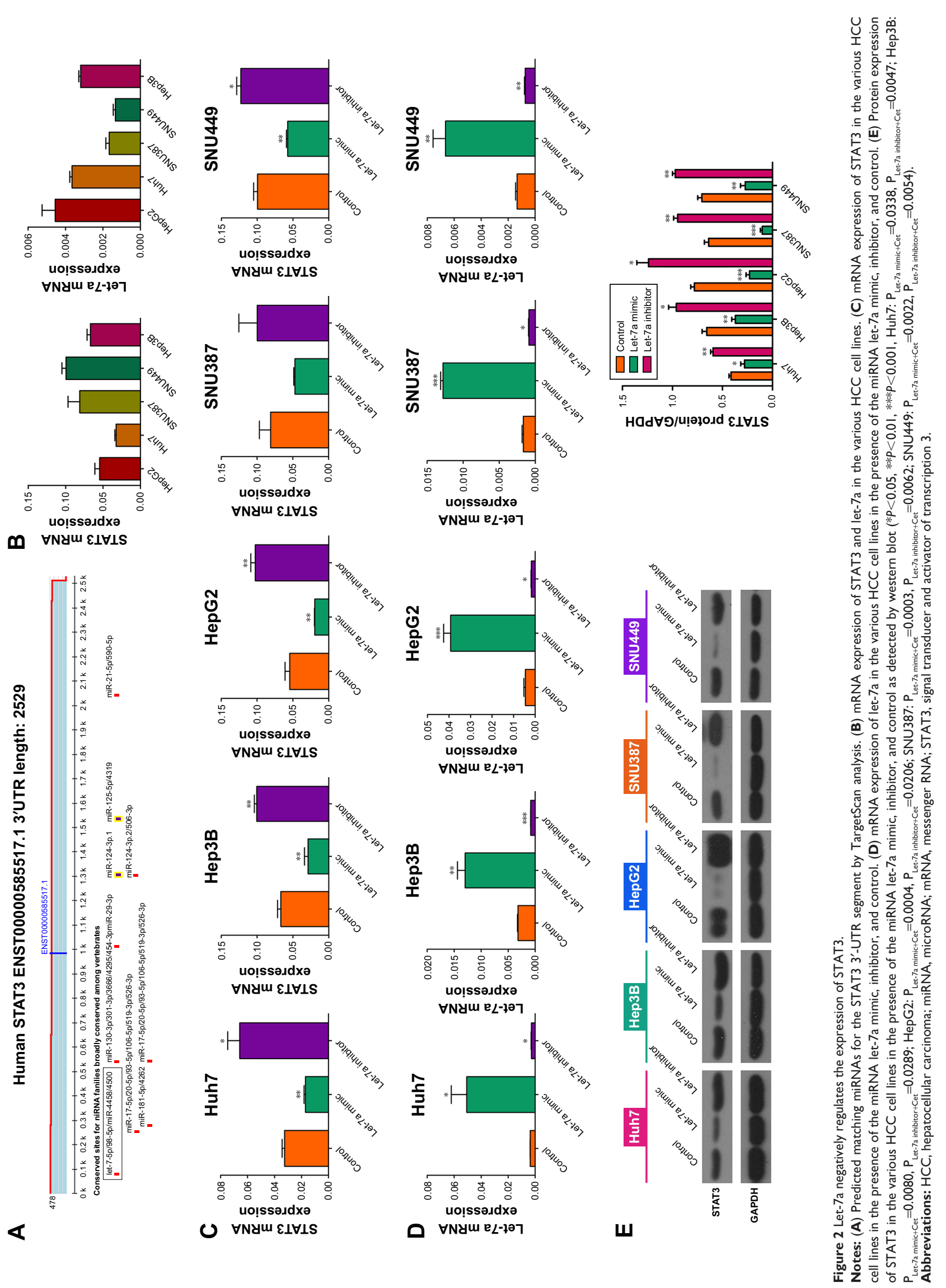
A
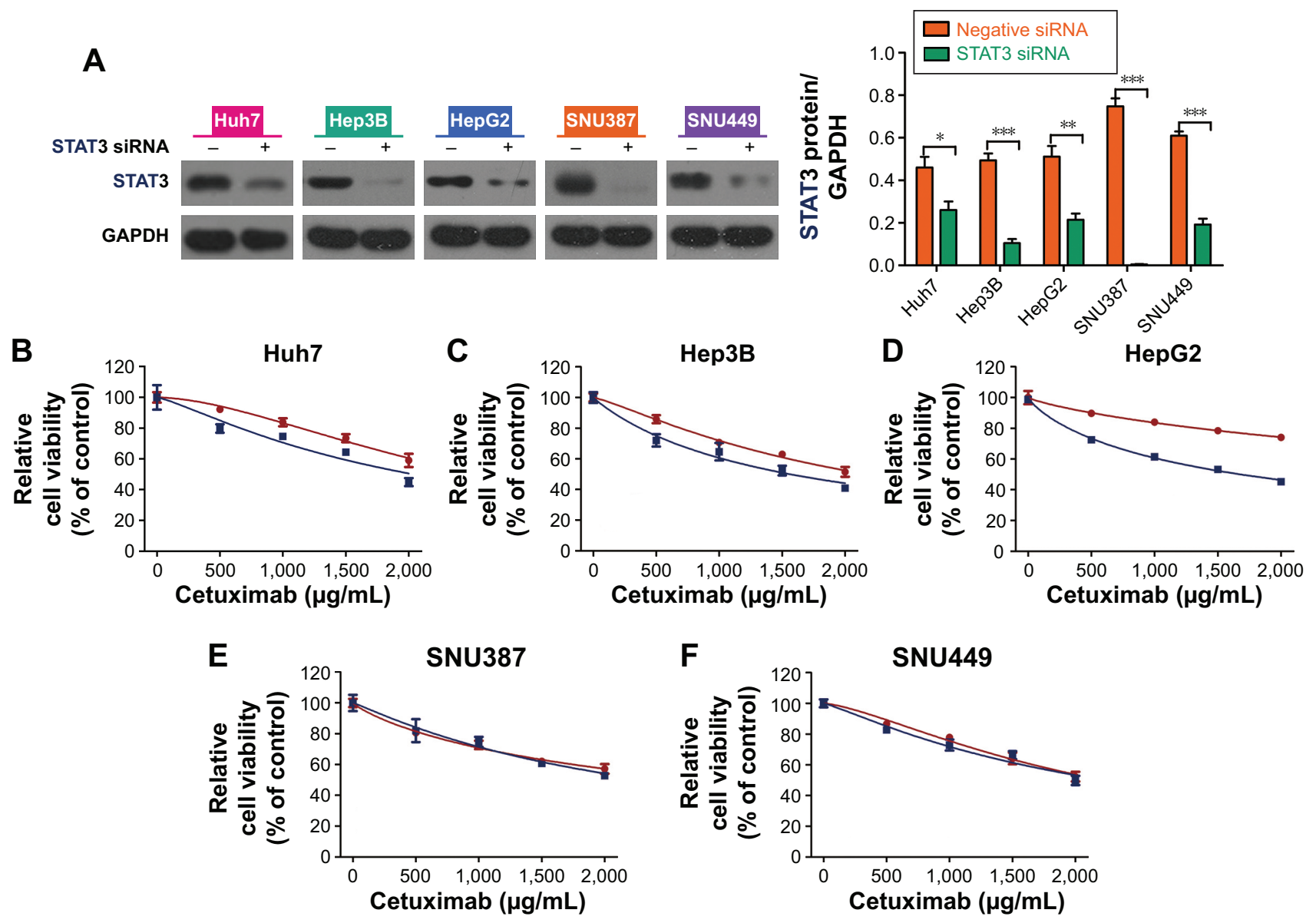

$\rightarrow$ Negative siRNA $\rightarrow$ STAT3 siRNA

Figure 3 Suppression of STAT3 could enhance the sensitivity of epithelial phenotype HCC cells to cetuximab.

Notes: (A) Transfection efficiency of STAT3 siRNA detected by Western blot in the five HCC cell lines; GAPDH was used as control $(* P<0.05$, $* * P<0.0$ I, ***P $<0.00$ I Huh7: $P=0.0353$; Hep3B: $P=0.0005$; HepG2: $P=0.0067$; SNU387: $P=0.000$ I; SNU449: $P=0.0002$ ). (B-F) Cell viability was determined using the CCK-8 assay in the presence of different concentrations of cetuximab $(0,500,1,000,1,500$, and 2,000 $\mu \mathrm{g} / \mathrm{mL})$. All HCC cell lines were separated into two groups: a STAT3 siRNA group and a negative siRNA control group.

Abbreviations: CCK-8, Cell Counting Kit-8; GAPDH, glyceraldehyde 3-phosphate dehydrogenase; HCC, hepatocellular carcinoma; siRNA, small interfering RNA; STAT3, signal transducer and activator of transcription 3.

Table $2 I_{50}$ values for cetuximab in $\mathrm{HCC}$ cell lines with negative siRNA plus cetuximab treatment or cetuximab plus STAT3 siRNA treatment

\begin{tabular}{lll}
\hline HCC cell line & $I C_{50}(\mu \mathrm{g} / \mathrm{mL})$ & \\
\cline { 2 - 3 } & $\begin{array}{l}\text { Negative } \\
\text { siRNA + cetuximab }\end{array}$ & $\begin{array}{l}\text { STAT3 } \\
\text { siRNA + cetuximab }\end{array}$ \\
\hline Huh7 & $2,564 \pm 109.8$ & $2,037 \pm 134.7^{*}$ \\
Hep3B & $2,167 \pm 68.96$ & $1,562 \pm 74.38^{*}$ \\
HepG2 & $7,167 \pm 2,293$ & $1,680 \pm 79.0 I^{*}$ \\
SNU387 & $2,554 \pm 154.2$ & $2,487 \pm 272.2$ \\
SNU449 & $2,220 \pm 71.31$ & $2,239 \pm 115.1$ \\
\hline
\end{tabular}

Notes: $* P<0.05$ vs negative siRNA + cetuximab. Data presented as mean \pm SD. Abbreviations: $\mathrm{HCC}$, hepatocellular carcinoma; $\mathrm{IC}_{50}$, half maximal inhibitory concentration; SD, standard deviation; siRNA, small interfering RNA; STAT3, signal transducer and activator of transcription 3. was decreased. To further verify our hypothesis, we used STAT3 siRNA to knockdown STAT3 expression in HCC cell lines. When the HCC cell lines were incubated in the presence of a control, a let-7a mimic, and a let-7a inhibitor, we found no differences in the sensitivity (cytoxicity) of the cells to cetuximab.

In conclusion, our study found that let-7a can enhance the sensitivity of HCCs with an epithelial phenotype to cetuximab by regulating STAT3 expression, but has no effect on HCC cells with the mesenchymal phenotype. This finding suggests a crucial role of let-7a in future miRNA-based combined chemotherapy. However, the validity and security 

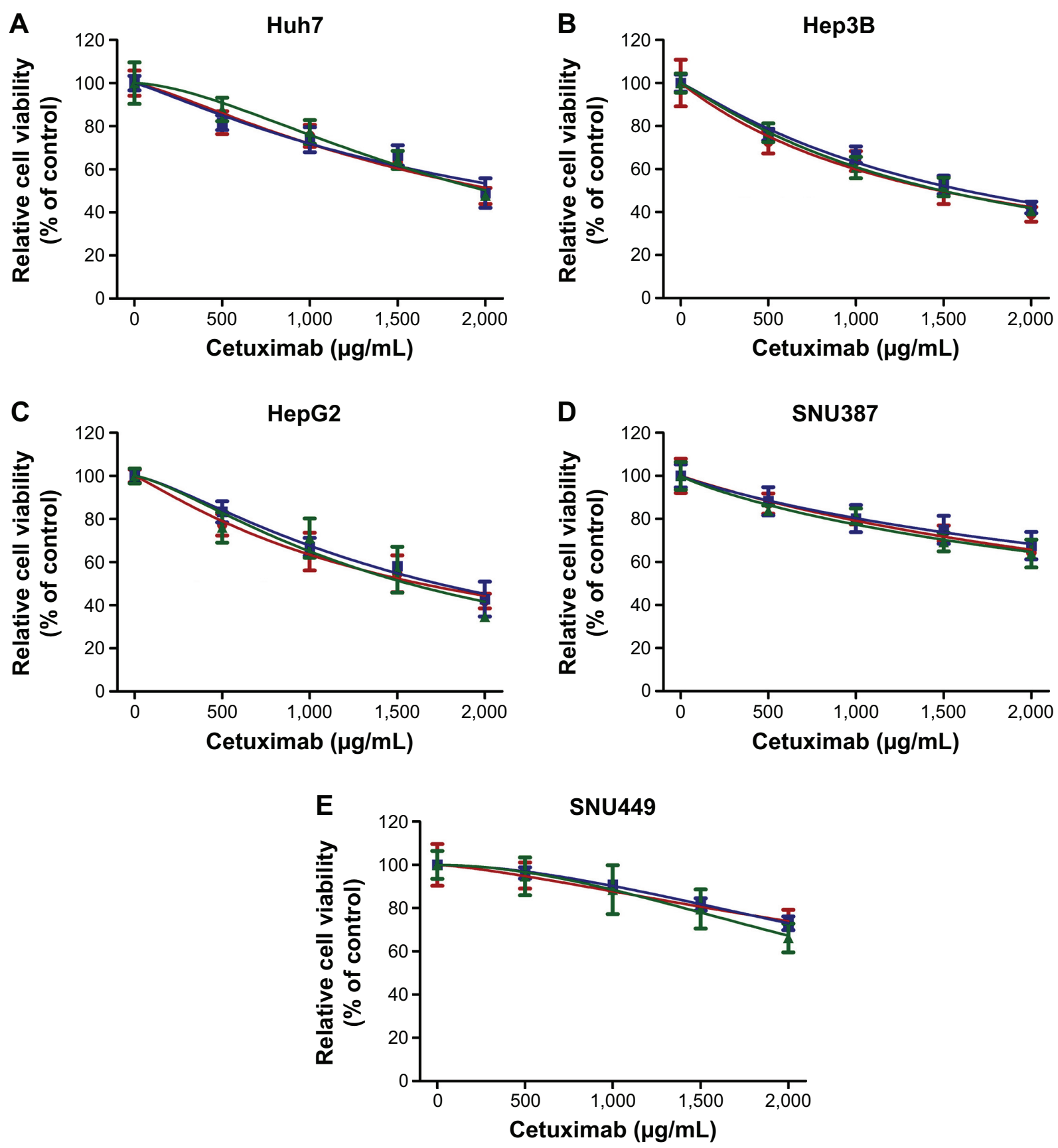

$\rightarrow$ Control $\rightarrow$ Let-7a mimic $\rightarrow$ Let-7a inhibitor

Figure 4 The role of let-7a on increasing the sensitivity of HCC cells to cetuximab is impaired after STAT3 suppression.

Notes: (A-E) After STAT3 knockdown, the HCC cell lines were separated into three groups, and separately transfected with the let-7a mimic, inhibitor, or control. Cell viability was determined using the CCK-8 assay in the presence of different concentrations of cetuximab $(0,500,1,000,1,500$, and 2,000 $\mu \mathrm{g} / \mathrm{mL})$.

Abbreviations: CCK-8, cell counting kit-8; HCC, hepatocellular carcinoma; STAT3, signal transducer and activator of transcription 3.

of combined chemotherapy based on let-7a should be carefully evaluated in well-designed clinical trials.

\section{Acknowledgments}

We would like to thank the native English speaking scientists of Elixigen Company (Huntington Beach, CA, USA) for editing our manuscript. This work was supported by grants from the National Natural Science Foundation of People's Republic of China (number 81273260) and the Henan provincial key scientific and technological project (number 132102310088).

\section{Disclosure}

The authors report no conflicts of interest in this work. 


\section{References}

1. Finn RS. Current and future treatment strategies for patients with advanced hepatocellular carcinoma: role of mTOR inhibition. Liver Cancer. 2012;1(3-4):247-256.

2. Zhou Y, Liang C, Xue F, et al. Salinomycin decreases doxorubicin resistance in hepatocellular carcinoma cells by inhibiting the $\beta$-catenin/TCF complex association via FOXO3a activation. Oncotarget. 2015;6(12): 10350-10365.

3. Forner A, Gilabert M, Bruix J, Raoul JL. Treatment of intermediatestage hepatocellular carcinoma. Nat Rev Clin Oncol. 2014;11(9): 525-535.

4. Wu Q, Yang Z, Nile Y, Shi Y, Fan D. Multi-drug resistance in cancer chemotherapeutics: mechanisms and lab approaches. Cancer Lett. 2014; 347(2):159-166.

5. Pirker R, Pereira JR, von Pawel J, et al. EGFR expression as a predictor of survival for first-line chemotherapy plus cetuximab in patients with advanced non-small-cell lung cancer: analysis of data from the phase 3 FLEX study. Lancet Oncol. 2012;13(1):33-42.

6. Zhu AX, Stuart K, Blaszkowsky LS, et al. Phase 2 study of cetuximab in patients with advanced hepatocellular carcinoma. Cancer. 2007;110(3): 581-589.

7. Sanoff HK, Bernard S, Goldberg RM, et al. Phase II study of capecitabine, oxaliplatin, and cetuximab for advanced hepatocellular carcinoma. Gastrointest Cancer Res. 2011;4(3):78-83.

8. Chen W, Hu QD, Xia XF, et al. Rapamycin enhances cetuximab cytotoxicity by inhibiting mTOR-mediated drug resistance in mesenchymal hepatoma cells. Cancer Biol Ther. 2014;15(8):992-999.

9. Huang S, He R, Rong M, Dang Y, Chen G. Synergistic effect of MiR-146a mimic and cetuximab on hepatocellular carcinoma cells. Biomed Res Int. 2014;2014:384121.

10. Augello C, Vaira V, Caruso L, et al. MicroRNA profiling of hepatocarcinogenesis identifies C19MC cluster as a novel prognostic biomarker in hepatocellular carcinoma. Liver Int. 2012;32(5):772-782.

11. Sato F, Hatano E, Kitamura K, et al. MicroRNA profile predicts recurrence after resection in patients with hepatocellular carcinoma within the Milan Criteria. PLoS One. 2011;6(1):e16435.

12. Liu Q, Lv GD, Qin X, et al. Role of microRNA let-7 and effect to HMGA2 in esophageal squamous cell carcinoma. Mol Biol Rep. 2012; 39(2):1239-1246.
13. Wagner S, Ngezahayo A, Murua Escobar H, Nolte I. Role of miRNA let-7 and its major targets in prostate cancer. Biomed Res Int. 2014;2014:376326.

14. Wong TS, Man OY, Tsang CM, et al. MicroRNA let-7 suppresses nasopharyngeal carcinoma cells proliferation through downregulating c-Myc expression. J Cancer Res Clin Oncol. 2011;137(3):415-422.

15. Guan $H$, Zhang $P$, Liu C, et al. Characterization and functional analysis of the human microRNA let-7a2 promoter in lung cancer A549 cell lines. Mol Biol Rep. 2011;38(8):5327-5334.

16. Li Z, Guo Y, Zhou L, et al. Association of a functional RAD52 genetic variant locating in a miRNA binding site with risk of HBV-related hepatocellular carcinoma. Mol Carcinog. 2015;54(9):853-858.

17. Roush S, Slack FJ. The let-7 family of microRNAs. Trends Cell Biol. 2008;18(10):505-516.

18. Brueckner B, Stresemann C, Kuner R, et al. The human let-7a-3 locus contains an epigenetically regulated microRNA gene with oncogenic function. Cancer Res. 2007;67(4):1419-1423.

19. Lu L, Katsaros D, de la Longrais IA, Sochirca O, Yu H. Hypermethylation of let-7a-3 in epithelial ovarian cancer is associated with low insulin-like growth factor-II expression and favorable prognosis. Cancer Res. 2007;67(21):10117-10122.

20. Ezzoukhry Z, Louandre C, Trecherel E, et al. EGFR activation is a potential determinant of primary resistance of hepatocellular carcinoma cells to sorafenib. Int J Cancer. 2012;131(12):2961-2969.

21. Ung N, Putoczki TL, Stylli SS, et al. Anti-EGFR therapeutic efficacy correlates directly with inhibition of STAT3 activity. Cancer Biol Ther. 2014;15(5):623-632.

22. Chen W, Shen X, Xia X, et al. NSC 74859-mediated inhibition of STAT3 enhances the anti-proliferative activity of cetuximab in hepatocellular carcinoma. Liver Int. 2012;32(1):70-77.

23. Wheeler SE, Suzuki S, Thomas SM, et al. Epidermal growth factor receptor variant III mediates head and neck cancer cell invasion via STAT3 activation. Oncogene. 2010;29(37):5135-5145.

24. Iida M, Brand TM, Starr MM, et al. Overcoming acquired resistance to cetuximab by dual targeting HER family receptors with antibody-based therapy. Mol Cancer. 2014;13:242.
OncoTargets and Therapy

\section{Publish your work in this journal}

OncoTargets and Therapy is an international, peer-reviewed, open access journal focusing on the pathological basis of all cancers, potential targets for therapy and treatment protocols employed to improve the management of cancer patients. The journal also focuses on the impact of management programs and new therapeutic agents and protocols on

\section{Dovepress}

patient perspectives such as quality of life, adherence and satisfaction. The manuscript management system is completely online and includes a very quick and fair peer-review system, which is all easy to use. Visit http://www.dovepress.com/testimonials.php to read real quotes from published authors. 